\title{
Asymmetric effects of speaking rate on voice-onset time: The case of Russian
}

\author{
Mayuki Matsui \\ Department of Linguistics, Hiroshima University, Japan \\ https://doi.org/10.36505/ExLing-2012/05/0022/000228
}

\begin{abstract}
Cumulative cross-linguistic studies have revealed asymmetries regarding the effects of speaking rate on voice-onset time of stop consonants. This paper reports the effects of speaking rate on VOT of lenis and fortis stops in Russian, an area of enquiry that has not been reported in previous literature. The overall results of the acoustic study indicated that lenis stops (pre-voiced VOT category) were greatly affected by changes in speaking rate, whereas fortis stops (short-lag VOT category) were only minimally affected. These results were consistent with those found for various other languages. The results also indicated that VOT distribution of Russian lenis and fortis categories only slightly overlap, even under the changes in speaking rate.
\end{abstract}

Key words: phonetics, phonology, speaking rate, voice-onset time, Russian

\section{Introduction}

This paper reports on the effects of speaking rate on voice-onset time in Russian.

Voice-onset time (henceforth, VOT) refers to a time difference between the release of oral closure and the onset of the vocal fold vibration. The phonological contrast of voicing in stop consonants is typically associated with VOT in many languages (e.g., Lisker and Abramson 1964). Since the value of VOT is defined by two independent temporal events, one question is how VOT is affected by the changes in global temporal properties, i.e. speaking rate. A considerable number of studies have addressed this question in languages such as English, French, Thai, and Spanish (e.g., Miller et al. 1986; Kessinger and Blumstein 1997; Magloire and Green 1999), and have found that speaking rate modifies VOTs in an asymmetric way: pre-voiced and long-lag (aspirating) VOT categories significantly change as a function of speaking rate, whereas the short-lag category remains roughly stable.

This paper presents the results for Russian, a language that has a two-way laryngeal contrast, typically realized as a pre-voiced vs. short-lag VOT (Ringen and Kulikov to appear). Following Ringen and Kulikov (to appear), this paper uses the term lenis and fortis for the pre-voiced and the short-lag category.

ExLing 2012: Proceedings of 5th Tutorial and Research Workshop on Experimental Linguistics, 27-29 August 2012, Athens, Greece 


\section{Methods}

Seven native speakers of Russian participated in a production study (four females and three males, mean age: 27.9). All of the speakers were born and raised around Orenburg city in the Russian Federation. Bilabial (/p/, /b/), dental $(/ \mathrm{t} /, / \mathrm{d} /)$, and velar $(/ \mathrm{k} /, / \mathrm{g} /)$ unpalatalized stops were examined. The vowels following the critical stops were $/ \mathrm{a} /, / \mathrm{o} /$, or $/ \mathrm{u} /$. Nine minimal or nearminimal pairs of monosyllabic real words with 18 fillers were presented to the speakers in a pseudo-random order. For our purpose of manipulating speaking rate, the speakers were asked to produce the target items in three conditions. In the first condition, the items were presented in isolation. In the second, they were embedded in a career sentence such as 'Skazhi, pozhalujsta, __ jesshe raz. (Please say ___ once again.)'. Finally, the item was presented again in the same career sentence, but the speakers were instructed to produce it quicker than the previous one.

The second and third conditions are defined as 'Slow' and 'Fast', respectively, and the words produced in these conditions were acoustically analysed by using the Praat speech analysis software (Boersma and Weenink 2010). In addition to VOT, word duration was measured as an index of speaking rate. Six tokens $(2.4 \%)$ were excluded due to the release burst and/or the word boundary not being clear. As a result, the remaining 246 tokens were submitted for further analyses.

\section{Results}

Firstly, paired t-tests confirmed that, for each speaker, word duration in the Slow condition was significantly longer than that in the Fast condition ( $p<$ 0.05 , or better), indicating that the rate manipulation proved successful.

Focusing on our primary question regarding the rate effects, as a first approximation, the overall distribution of VOT are illustrated in Figure 1. As seen in Figure 1, lenis and fortis stops were typically realized as pre-voiced and short-lag VOTs, respectively, replicating Ringen and Kulikov (to appear). Figure 1 also shows that there was little overlap between the two categories.

Next, in order to examine the effects of speaking rate, $2 \times 3$ (Rate Condition $\times$ Place of Articulation) repeated-measure ANOVAs were conducted on VOTs for lenis and fortis stops separately. ANOVA was applied first for the overall tokens, and then for part of the tokens, where the vowel following the critical stop was controlled. Here, the results for the latter case are reported. The mean values and standard deviations are summarized in table 1. 


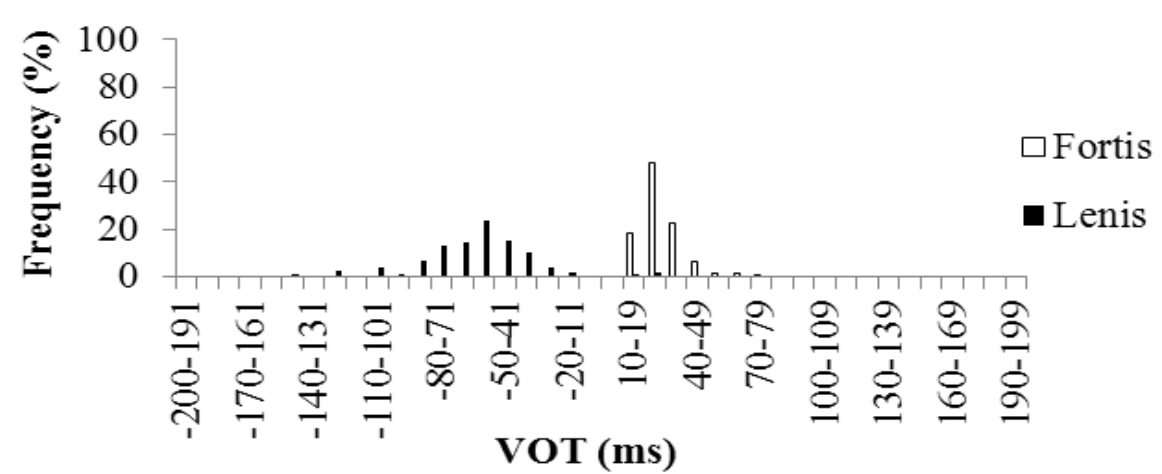

Figure 1. The overall VOT distribution of lenis and fortis stops. (10-ms bin).

Table 1. Mean VOT values where the following vowel was fixed as $/ \mathrm{o} /$ Standard deviations are indicated in parenthesis.

\begin{tabular}{llllllll}
\hline & \multicolumn{2}{l}{ Lenis stops } & & \multicolumn{3}{l}{ Fortis stops } \\
\cline { 2 - 3 } \cline { 7 - 8 } & Slow & Fast & Diff. & & Slow & Fast & Diff. \\
\hline Bilabial & $-90(23)$ & $-63(15)$ & 27 & & $17(8)$ & $12(4)$ & 5 \\
Dental & $-87(35)$ & $-63(19)$ & 24 & & $12(5)$ & $13(6)$ & -1 \\
Velar & $-69(22)$ & $-54(15)$ & 15 & & $30(9)$ & $22(8)$ & 8 \\
\hline
\end{tabular}

The results showed that, for lenis stops, a significant main effect of Rate Condition was found $(F(1,5)=9.319, p<0.05)$, but neither significant main effect of Place of Articulation $(F(2,10)=1.638, p=0.243)$, nor interaction $(F(2,10)=0.880, p=0.445)$ was observed. For fortis stops, the main effect of Rate was marginal $(F(1,5)=5.770, p=0.061)$. There was a significant main effect of Place $(F(2,10)=17.456, p<0.01)$, with velar being longer than dental $(p<0.01)$. No significant interaction was found $(F(2,10)=2.064$, $p=0.178)$.

Finally, a linear function relating VOT to word duration was estimated. Taking potential speaker-specific differences into account (See Theodore et al. 2009), a hierarchical linear modelling (HLM) analysis was applied for lenis and fortis stops separately. In each model, VOT values were nested within the seven speakers. The HLM analysis obtained a mean slope of -0.14 $\mathrm{ms}$ for lenis stops and $0.09 \mathrm{~ms}$ for fortis stops. Both slopes were significantly greater than zero $\left(t_{\text {lenis }}(6)=-2.998, p<0.05 ; t_{\text {fortis }}(6)=5.029, p<0.01\right)$.

To summarize, the results of ANOVA and HLM analyses showed that both lenis and fortis stops were more or less affected by changes in speaking rate. However, fortis stops showed little change across speaking rate, suggesting that speaking rate has asymmetric effects on VOT. 


\section{Discussion and conclusion}

This study examined the effects of speaking rate on VOT in the Russian language. The results indicated asymmetric effects of speaking rate, that is, fortis (short-lag) stops were less affected by changes in speaking rate. The results are consistent with those reported for various other languages. The results also showed that under the changes in speaking rate, there was little overlap between lenis and fortis distributions along the VOT continuum.

It is interesting to note that the correlation between distributional overlap of VOT and perceptual shift as a function of speaking rate has been suggested for English, which has a considerable overlap in VOT distribution (Miller et al. 1986). On the other hand, in languages with little overlap such as Russian, the effects of speaking rate on the perception of voicing have not been investigated. Future studies could investigate the perception of voicing under changes of speaking rate in languages with little overlap, which may play a role in forming a typology of the correlation between production and perception as a function of speaking rate.

\section{Acknowledgements}

The author would like to thank Liudmila Dokashenko for her help with carrying out the experiment at Orenburg State University. This research was partly supported by Grant-in-Aid for JSPS Fellows (\#246727).

\section{References}

Boersma, P., Weenink, D. 2010. Praat: Doing phonetics by computer (Version 5.1.31) [computer program]. Retrieved April 4, 2010, <from http://www.praat.org/>

Kessinger, R.H., Blumstein, S.E. 1997. Effects of speaking rate on voice-onset time in Thai, French, and English. Journal of Phonetics 25, 143-168.

Lisker, L., Abramson, A.S. 1964. Cross-language study of voicing in initial stops: acoustical measurements. Word 20, 384-422.

Magloire, J., Green, K.P. 1999. A Cross-language comparison of speaking rate effects on the production of voice onset time in English and Spanish. Phonetica $56,158-185$.

Miller., J.L., Green, K.P., Reeves., A. 1986. Speaking rate and segments: A look at the relation between speech production and speech perception for the voicing contrast. Phoneica 43, 106-115.

Ringen, C., Kulikov, V. To appear. Voicing in Russian stops: Cross-linguistic implications. Journal of Slavic Linguistics.

Theodore, R.M., Miller, J.L., DeSteno, D. 2009. Individual talker differences in voice-onset time: Contextual influences. Journal of the Acoustical Society of America 125, 3974-3982. 